\title{
Genomic Analysis of Early Transmissibility Assessment of the D614G Mutant Strain of SARS-CoV-2 in Travelers Returning to Taiwan from the United States of America
}

\author{
Ming-Jr Jian ${ }^{1}$, Hsing-Yi Chung ${ }^{1}$, Chih-Kai Chang ${ }^{1}$, Shan-Shan Hsieh ${ }^{1}$, Jung-Chung Lin ${ }^{2}$, Kuo-Ming Yeh ${ }^{2}$, Chien- \\ Wen Chen ${ }^{3}$, Feng-Yee Chang ${ }^{2}$, Kuo-Sheng Hung ${ }^{4}$, Ming-Tsan Liu ${ }^{5}$, Ji-Rong Yang ${ }^{5}$, Tein-Yao Chang ${ }^{6}$, Sheng-Hui \\ Tang ${ }^{1}$, Cherng-Lih Perng ${ }^{1}$, Hung-Sheng Shang ${ }^{\text {Corresp. } 1}$ \\ ${ }^{1}$ Division of Clinical Pathology, Department of Pathology, Tri-Service General Hospital, National Defense Medical Center, Taipei, Taiwan, Taipei city, \\ Taiwan \\ 2 Division of Infectious Diseases and Tropical Medicine, Department of Medicine, Tri-Service General Hospital, National Defense Medical Center, Taipei, \\ Taiwan, Taipei city, Taiwan \\ 3 Division of Pulmonary and Critical Care Medicine, Department of Medicine, Tri-Service General Hospital, National Defense Medical Center, Taipei, \\ Taiwan, Taipei city, Taiwan \\ 4 Center for Precision Medicine and Genomics, Tri-Service General Hospital, National Defense Medical Center, Taipei, Taiwan, Taipei city, Taiwan \\ 5 Centers for Disease Control, Taipei, Taiwan, Taipei city, Taiwan \\ 6 Institute of Preventive Medicine, National Defense Medical Center, Taipei city, Taiwan \\ Corresponding Author: Hung-Sheng Shang \\ Email address: iamkeith001@gmail.com
}

\begin{abstract}
Background: There is a global pandemic of severe acute respiratory syndrome coronavirus 2 (SARS-CoV-2). Information on viral genomics is crucial for understanding global dispersion and for providing insight into viral pathogenicity and transmission. Here, we characterized the SARS-CoV-2 genomes isolated from five travelers who returned to Taiwan from the United States of America (USA) in March and June 2020.

Methods: Haplotype network analysis was performed using genome-wide single-nucleotide variations to trace potential infection routes. To determine the genetic variations and evolutionary trajectory of the isolates, the genomes of isolates were compared to those of global virus strains from GISAID. Pharyngeal specimens were confirmed to be SARS-CoV-2-positive by RT-PCR. Direct whole-genome sequencing was performed, and viral assemblies were subsequently uploaded to GISAID. Comparative genome sequence and single-nucleotide variation analyses were performed.

Results: The D614G mutation was identified in imported cases, which separated into two clusters related to viruses originally detected in the USA. Our findings highlight the risk of spreading SARS-CoV-2 variants through air travel and the need for continued genomic tracing for the epidemiological investigation and surveillance of SARS-CoV-2 using viral genomic data.
\end{abstract}

Conclusions: Continuous genomic surveillance is warranted to trace virus circulation and evolution in different global settings during future outbreaks. 
2 CoV-2 in Travelers Returning to Taiwan from the United States of America

3 Ming-Jr Jian ${ }^{\text {a }}$, Hsing-Yi Chung ${ }^{\text {a }}$, Chih-Kai Changa, Shan-Shan Hsieh ${ }^{\text {a }}$, Jung-Chung Lin ${ }^{\text {, }}$, Kuo-

4 Ming Yeh ${ }^{\mathrm{b}}$, Chien-Wen Chen ${ }^{\mathrm{c}}$, Feng-Yee Chang ${ }^{\mathrm{b}}$, Kuo-Sheng Hung ${ }^{\mathrm{d}}$, Ming-Tsan Liu ${ }^{\mathrm{e}}$, Ji-Rong

5 Yang ${ }^{\mathrm{e}}$, Tein-Yao Chang, Sheng-Hui Tanga ${ }^{\mathrm{a}}$, Cherng-Lih Perng ${ }^{\mathrm{a}}$ and Hung-Sheng Shang**

6 aDivision of Clinical Pathology, Department of Pathology, Tri-Service General Hospital, National

7 Defense Medical Center, Taipei, Taiwan, ROC

8 bivision of Infectious Diseases and Tropical Medicine, Department of Medicine, Tri-Service General

9 Hospital, National Defense Medical Center, Taipei, Taiwan, ROC ${ }^{\mathrm{c} D i v i s i o n}$ of Pulmonary and Critical

10 Care Medicine, Department of Medicine, Tri-Service General Hospital, National Defense Medical Center,

11 Taipei, Taiwan, ROC ${ }^{\mathrm{d} C e n t e r}$ for Precision Medicine and Genomics, Tri-Service General Hospital,

12 National Defense Medical Center, Taipei, Taiwan, ROC; ${ }^{\mathrm{e} C e n t e r s ~ f o r ~ D i s e a s e ~ C o n t r o l, ~ T a i p e i, ~ T a i w a n, ~}$

13 ROC

14 Institute of Preventive Medicine, National Defense Medical Center, ROC

* Correspondence: Hung-Sheng Shang; Division of Clinical Pathology, Department of Pathology, Tri-

17 Service General Hospital, National Defense Medical Center, 325, Section 2, Cheng-Kung Road, Neihu

18 114, Taipei, Taiwan, Republic of China; Tel +886920713130; Fax +886287927226; Email

19 iamkeith001@gmail.com 


\section{Abstract:}

22 Background: There is a global pandemic of severe acute respiratory syndrome coronavirus 2 (SARS-

$23 \mathrm{CoV}-2)$. Information on viral genomics is crucial for understanding global dispersion and for providing

24 insight into viral pathogenicity and transmission. Here, we characterized the SARS-CoV-2 genomes

25 isolated from five travelers who returned to Taiwan from the United States of America (USA) in March

26 and June 2020.

27 Methods: Haplotype network analysis was performed using genome-wide single-nucleotide variations to

28 trace potential infection routes. To determine the genetic variations and evolutionary trajectory of the

29 isolates, the genomes of isolates were compared to those of global virus strains from GISAID. Pharyngeal

30 specimens were confirmed to be SARS-CoV-2-positive by RT-PCR. Direct whole-genome sequencing

31 was performed, and viral assemblies were subsequently uploaded to GISAID. Comparative genome

32 sequence and single-nucleotide variation analyses were performed.

33 Results: The D614G mutation was identified in imported cases, which separated into two clusters related

34 to viruses originally detected in the USA. Our findings highlight the risk of spreading SARS-CoV-2

35 variants through air travel and the need for continued genomic tracing for the epidemiological

36 investigation and surveillance of SARS-CoV-2 using viral genomic data.

37 Conclusions: Continuous genomic surveillance is warranted to trace virus circulation and evolution in

38 different global settings during future outbreaks.

39 Keywords: COVID-19; SARS-CoV-2; whole-genome sequencing; phylogenetic analysis; imported case 


\section{Introduction}

Severe acute respiratory syndrome coronavirus 2 (SARS-CoV-2) is the seventh coronavirus known to infect humans. Among them, SARS-CoV, MERS-CoV, and SARS-CoV-2 can cause severe disease, whereas HKU1, NL63, OC43, and 229E are associated with mild symptoms (Andersen et al., 2020; Gralinski and Menachery, 2020; Xie and Chen, 2020). SARS-CoV-2 is the etiological cause of coronavirus disease 2019 (COVID-19), which was first identified in humans in China in late December 2019 (Wu and McGoogan, 2020). The outbreak has since spread further and as of March 2021, there have been 100 million confirmed cases of COVID-19 worldwide with over 2 million deaths (https://www.who.int/publications/m/item/covid-19-weekly-epidemiological-update; access date: 2021/03/08). Fewer than 1000 cases of COVID-19 have been reported on the self-governing island of Taiwan (through March 8, 2021), a number that has remained relatively low due to a series of aggressive containment, quarantine, and monitoring measures that have limited local SARS-CoV-2 transmission (Chang, 2020; Chen et al., 2020; Cheng et al., 2020). The majority of confirmed COVID-19 cases were imported from other countries, including America, European countries, and other Asian countries (Lin et al. 2020).

Mutations arise as a natural occurrence of viral replication. When a newly arising mutation confers a competitive advantage with respect to viral replication, transmission, or escape from immunity, that mutation is maintained in the overall virus population (Andersen et al., 2020; Uddin et al., 2020). Several SARS-CoV-2 genes have been found to evolve, including those encoding the nucleocapsid $(\mathrm{N})$, viral replicase, and spike proteins (S) (Dilucca et al., 2020). The D614G mutation in the spike glycoprotein of SARS-CoV-2 was first detected at a significant level in early March 2020 and spread to global dominance over the following few months (Al-Zyoud and Haddad, 2021; Isabel et al., 2020,;Islam et al., 2020; Korber et al., 2020; Zhang et al., 2020). Up-to-date information on viral genomics is crucial for understanding the global dispersion of SARS-CoV-2 and for providing insight into its pathogenicity (Furuse, 2021; Guan et al., 2020). Knowing the genome sequence helps us understand how SARS-CoV-2 is mutating into variants and how the virus is passing between people. 
67 late February 2020, and the second from other countries starting in early March 2020(Gong et al. 2020;

68 Jian et al. 2021). To determine the genetic variations and evolutionary trajectory of the isolates, we

69 compared their genomes to those of global virus strains available through GISAID (Lo \& Jamrozy 2020).

70 Here, we characterized the SARS-CoV-2 genome sequences from five travelers who returned to Taiwan

71 from the United States of America during March and April, 2020. Genome sequences of SARS-CoV-2

72 were then compared with those deposited in GISAID in order to characterize the genealogical networks

73 and identify possible routes of transmission. Our findings may be used to show how variants of SARS-

74 CoV-2 entered Taiwan, and to determine their association with the implemented quarantine system for

75 entry measures established by the Central Epidemic Command Center (CECC), Taiwan. Our findings

76 have implications for epidemiological investigations and surveillance of viral genomic data.

\section{Materials and Methods}

Collection of Clinical Specimens

79 Nasopharyngeal swabs (COPAN's COVID-19 Collection \& Transport Kits with Universal Transport

80 Medium or Virus Transport Swabs 147C) were obtained from travelers suspected of having COVID-19.

81 This study was approved by the Institutional Review Board of Tri-Service General Hospital (TSGHIRB

82 No.: C202005041), and was registered on March 20, 2020. Written informed consent was obtained from

83 participants for publication of the case reports. The same IRB NO (TSGHIRB No.: C202005041) used in

84 a previous publication (Jian et al. 2021). We need to clarify the objective is sufficiently different and non-

85 overlapping which the previous publication aimed to investigate the clinical characteristics and

86 differentiation of genetic variation among isolates from a cluster of local familial COVID-19 infection. In

87 this study we addressed different questions with more complex analyses and focus on SARS-CoV-2

88 genome sequences from travelers who returned to Taiwan from the United States of America. The aim of

89 this study was to trace virus circulation and evolution in Taiwan. Since the D614G mutation in the spike 
90 glycoprotein of SARS-CoV-2 surged in early March 2020, variant cases were selected between March to

91 April 2020. Total nucleic acid containing viral RNA was extracted from $0.3 \mathrm{~mL}$ of the throat swab

92 supernatant using LabTurbo Viral nucleic acid extraction kits on a LabTurbo 48 AIO automatic extractor

93 (Taigen Bioscience Corp., Taipei, Taiwan). RNA was eluted with $60 \mu \mathrm{L}$ of RNase-free water. (Perng et

94 al. 2020).

95

96

97

98

SARS-CoV-2 Real-time Reverse Transcription-Polymerase Chain Reaction (RT-PCR) Testing

Pharyngeal specimens were collected from travelers with symptoms of suspected COVID-19. The presence of SARS-CoV-2 was confirmed by RT-PCR testing according to the guidelines of the Taiwan Center for Disease Control (CDC), as described previously (Jian et al., 2021). Briefly, a SARS-CoV-2 real-time RT-PCR assay using primers, probe, and RT-PCR reagents to detect the $R d R p$ and $E$ genes of SARS-CoV-2 was performed on an AIO 48 System (LabTurbo, New Taipei City, Taiwan). A diluted viral RNA sample from a COVID-19-positive patient was used as a positive control, which was aliquoted and stored at $-80^{\circ} \mathrm{C}$ (a Ct values of $34 \pm 2$ per run was considered acceptable) (Jian et al., 2021). All positive samples were confirmed by the Taiwan CDC Central Laboratory. The five cases (Cases 1-5) were obtained between March and June 2020 and included subjects with a travel history from the USA to Taiwan.

\section{Whole-Genome Sequencing of SARS-CoV-2}

The Ovation RNA-Seq System V2 (Nugen Technologies, San Carlos, CA, USA) was used to synthesize cDNA, which was then processed into a library described previously (Gong et al. 2020). WGS was performed as described previously (Jian et al. 2021). Briefly, whole-genome sequences of the SARSCoV-2 isolates (TSGH-04, TSGH-08, TSGH-22, TSGH-23, and TSGH-29) were obtained using the Illumina TruSeq Stranded mRNA Library Prep Kit protocol to enrich SARS-CoV-2 cDNA using multiplex RT-PCR amplicons. Next-generation sequencing (NGS) was performed on the NovaSeq 6000 platform (Illumina, San Diego, USA) with paired-end reads. 
114

115

116

117

118

119

120

121

122

123

124

125

126

127

128

129

130

131

132

133

134

135

136

137

\section{Phylogenetic Relationship Analysis}

Phylogenetic analysis was performed as described previously (Jian et al. 2021). Briefly, the SARSCoV-2 genome sequences from GISAID, and those of the five cases reported herein, were aligned using Clustal Omega (Sievers et al., 2011). Aligned nucleic acid sequences were then used to construct phylogenetic trees based on the neighbor-joining tree algorithms provided by PHYLIP (Felsenstein, 2005). Using the file created by PHYLIP, the tree was drawn and managed using MEGA X (Kumar et al., 2018). Bootstrap analysis was performed with 1000 replicates and the values were presented adjacent to the branch nodes (Felsenstein, 1985).

Comparative Genome Sequence Analysis and Single Nucleotide Variation (SNV) Analysis

The almost full-length genome sequence of SARS-CoV-2 $(\geq 29 \mathrm{~kb})$ was retrieved from the GISAID EpiCoV database on February 05, 2021, followed by multiple alignments using MAFFT v7.222. The core regions were compared with those of the Wuhan-Hu-1 genome sequences. The five genome sequences, together with sequences retrieved from GISAID, were aligned using MAFFT. SNV median-joining network analysis was performed using PopART software3 (http://popart.otago.ac.nz.), as previously described (Sekizuka et al., 2020). Briefly, early stage (2020/02/18-2020/06/19) SARS-CoV-2 genome sequence from USA were aligned to TSGH-04, TSGH-08, TSGH-22, TSGH-23, and TSGH-29. After further analysis of aligned sequences through CD-HIT, 129 sequences from the USA were selected to BLAST in GISAD, and 3,867 highly similar sequence were applied in a haplotype network analysis combined with COVID-19 sequences from TSGH-04, TSGH-08, TSGH-22, TSGH-23, TSGH-29.

\section{Results}

\section{Phylogenetic Relationship Analysis}

Data regarding the genome sequences of the five strains obtained in our study (Table 1) were deposited in the GISAID database. To investigate the genomic relationships of these five strains, and to identify the possible sources of infection, we used SARS-CoV-2 sequences from the USA, which spread

Peer] reviewing PDF | (2021:04:59830:2:1:NEW 25 Jul 2021) 
138 between 2020/02/18 and 2020/06/19, deposited in the GISAID database. These sequences were aligned to

139 those of TSGH-04, TSGH-08, TSGH-22, TSGH-23, and TSGH-29. The alignment results were further

140 clustered by CD-HIT. After cluster analysis, 129 sequences from the USA were selected and aligned. The

141 alignment was used to decipher the time-evolution relationship of the different SARS-CoV-2 genomes.

142 Our data showed that the five strains clustered in different positions of phylogenetic tree (Figure1). This

143 indicated that the five imported cases of COVID-19 were likely to have originated from contact with

144 different infection sources in the USA. Further characterization of genetic lineages class shows the five

145 SARS-CoV-2 sequences diversified into lineages B, with multiple sub-lineages B (TSGH-29), B1

146 (TSGH-04, TSGH-08, and TSGH-23), and B.1.369 (TSGH-22) confirmed by PANGOLIN software

147 (https://pangolin.cog-uk.io/) (Supplementary Table 1).

148

149

150

151

152

153

154

155

156

157

158

159

160

161

\section{Amino Acid Variation Mapping of SARS-CoV-2 Genomes and Network Analysis}

Analysis of the complete genomes of five SARS-CoV-2 strains from patients with a travel history to the USA revealed several missense mutations. The mutations occurred in the S-protein, non-structural protein, and open reading frames (ORFs) (Table 2), including the D614G variant in the S-protein in Cases 1-4; the wild-type form was identified in Case 5. In addition to the S-protein D614G mutation, other missense mutations were identified in NSP12 (RNA dependent RNA polymerase; RdRp) with a P378L mutation, a T85I mutation in NSP2, and a Q57H mutation in ORF3a protein. The genetic changes highlighted above are detailed in Table 2, based on the glue application (http://cov-glue.cvr.gla.ac.uk/) (Supplementary Table 2). To determine the timing of transmission routes for the five travelers who returned to Taiwan from the USA, whole-genome sequences of the isolates were compared by medianjoining SNV network analysis with those of all available SARS-CoV-2 genomes in the GISAID database ( $n=3872$; retrieved Aug 9, 2020). Our findings suggested that these current strains were related to, and clustered chronologically with, other globally identified viruses originally detected in the USA from December 2019 to May 2020 (Figure 2).

Peer) reviewing PDF | (2021:04:59830:2:1:NEW 25 Jul 2021) 


\section{Discussion}

163 Up to March 2021, there had been approximately 1,000 confirmed cases of COVID-19 in Taiwan.

164 Although the number of COVID-19 cases in Taiwan is low compared with other countries, we evaluated

165 five genome sequences of SARS-CoV-2 to understand viral evolution. To gain a better understanding of

166 the genomic epidemiology of the COVID-19 outbreak in Taiwan, we characterized the full genome

167 sequences of five SARS-CoV-2 strains collected from individuals traveling from the USA to Taiwan who

168 were diagnosed with COVID-19 and confirmed to be SARS-CoV-2-positive by RT-PCR. Then, we

169 performed a haplotype network analysis of the SARS-CoV-2 isolates using genome-wide single-

170 nucleotide variations. The D614G SARS-CoV-2 variant appears to have independently arisen and then

171 dispersed throughout multiple geographic regions, including Europe, Latin America, and Asia (Isabel et

172 al., 2020; Islam et al., 2020; Kannan et al., 2020). This is consistent with the timeline of the five COVID-

17319 cases we evaluated in the current study. Our analysis identified polymorphisms in the S-protein of the

174 current imported cases, which separated into two clusters that were related to globally detected viruses

175 first detected in the USA. Previous studies have shown that the P323L mutation in NSP12 co-evolved

176 with the D614G mutation in S-protein (Kannan et al., 2020; Vilar and Isom, 2021). Our current findings

177 are consistent with this observation, in that the viral genome sequences of the SARS-CoV-2 strains from

178 Cases 1-4 harbored both the D614G and P323L mutations. Case 5 was found to be wild type for these

179 mutations, and was collected before D614G had become the dominant variant in the world. This

180 demonstrates the need for public health bodies to respond to new viral genetic variants. This is also

181 substantiated by the emergence of the new "UK variant" (lineage B.1.1.7), which began circulating

182 between early September and mid-November, 2020 and has become a variant of widespread concern

183 (Lauring and Hodcroft, 2021; Leung et al., 2021). More stringent border control and quarantine

184 restrictions have been imposed in response to the emergence of new SARS-CoV-2 variants, such as the

185 UK variant. The spread of this new variant has led to a surge in COVID-19 cases and deaths (Davies et al.

186 2021). Enhanced genomic surveillance combined with increased compliance with public health mitigation

Peer] reviewing PDF | (2021:04:59830:2:1:NEW 25 Jul 2021) 
187 strategies, including vaccination, physical distancing, use of masks, hand hygiene, and isolation and 188 quarantine, will be essential to limiting the spread of SARS-CoV-2 and protecting public health (Lo \& 189 Jamrozy 2020).

190 The current study highlights the importance of genomic tracing of the SARS-CoV-2 genome as an 191 epidemiological tool and means of virus surveillance. Travelers have played a significant role in 192 introducing new cases of COVID-19 to countries, perpetuating ongoing human-to-human transmission 193 during the pandemic (Rodriguez-Morales et al., 2020). The high transmissibility of SARS-CoV-2, before 194 and immediately after the onset of COVID-19 symptoms, suggests that generalized protective measures 195 may be required, such as social distancing and the wearing of masks (Cheng et al., 2020). Taiwan was 196 expected to experience the second highest number of COVID-19 cases due to its proximity to the coast of 197 mainland China. This was avoided due to big data analysis and effective quarantine measures in 198 combination with strong public policies implemented by Taiwan's CECC and CDC. This included the 199 establishment of an entry quarantine system requiring travelers to complete a health declaration form either prior to departure or upon arrival at a Taiwan airport (Lin et al., 2020; Wang et al., 2020). Early assessment of airport entry screening has helped Taiwan to detect imported cases of COVID-19, 202 preventing the further spread of the pandemic.

203 Given the widespread transmission of SARS-CoV-2, additional sequence data regarding new strains 204 are required to fully understand how the virus has spread and evolved. Understanding SARS-CoV-2 205 variants remains an issue of concern for all governments, and is critical for establishing and implementing 206 effective public health measures. Genomic surveillance of COVID-19 can generate meaningful 207 information for tracking SARS-CoV-2 transmission, and is useful for the real-time response of health 208 departments. 
210 Funding: This study was supported by the Tri-Service General Hospital, Taipei, Taiwan, ROC (grant

211 number: TSGH-D-110100). The funders had no role in study design, data collection, or interpretation, nor

212 in the decision to submit the work for publication.

213 Conflicts of Interest: The authors report no conflicts of interest associated with this work.

214

215 Author Contributions: Conceptualization, Jung-Chung Lin, Kuo-Ming Ye, Chien-Wen Chen, and Feng-

216 Yee Chang; data curation, Ming-Jr Jian, Hsing-Yi Chung, and Kuo-Sheng Hung; formal analysis, Hung-

217 Sheng Shang and Ming-Jr Jian; investigation, Hsing-Yi Chung, Chih-Kai Chang, Ming-Tsan Liu, Ji-Rong

218 Yang, and Cherng-Lih Perng; methodology, Chih-Kai Chang, Shan-Shan Hsieh, and Cherng-Lih Perng;

219 resources, Hung-Sheng Shang, Kuo-Sheng Hung, and Tein-Yao Chang; Software, Kuo-Sheng Hung;

220 supervision, Hung-Sheng Shang, Jung-Chung Lin, Kuo-Ming Ye, Chien-Wen Chen, Feng-Yee Chang,,

221 Ji-Rong Yang, Sheng-Hui Tang, and Cherng-Lih Perng; validation, Ming-Tsan Liu; Writing - original

222 draft, Ming-Jr Jian; Writing - review \& editing, Hung-Sheng Shang. 


\section{References}

225 Al-Zyoud W, Haddad H. 2021. Mutational sensitivity of D614G in spike protein of SARS-CoV-2 in 226 Jordan. Biochemistry Biophysics Reports 25:100896.

227 Andersen KG, Rambaut A, Lipkin WI, Holmes EC, Garry RF. 2020. The proximal origin of SARS-CoV228 2. Nature Medicine 26:450-452.

229 Chang W-H. 2020. The influences of the COVID-19 pandemic on medical service behaviors. Taiwanese $230 \quad$ Journal of Obstetrics and Gynecology 59:821-827.

231 Chen C-C, Tseng C-Y, Choi W-M, Lee Y-C, Su T-H, Hsieh C-Y, Chang C-M, Weng S-L, Liu P-H, Tai 232 Y-L, and Lin C-Y. 2020. Taiwan Government-Guided Strategies Contributed to Combating and 233 Controlling COVID-19 Pandemic. Frontiers in Public Health 8:547423. 10.3389/fpubh.2020.547423 PMID - 33194949

235

236

237

238

239

Cheng H-Y, Jian S-W, Liu D-P, Ng T-C, Huang W-T, Lin H-H, and Team TC-OI. 2020. Contact Tracing Assessment of COVID-19 Transmission Dynamics in Taiwan and Risk at Different Exposure Periods Before and After Symptom Onset. JAMA Internal Medicine 180:1156-1163. 10.1001/jamainternmed.2020.2020 PMID - 32356867 Dilucca M, Forcelloni S, Georgakilas AG, Giansanti A, Pavlopoulou A. 2020. Codon Usage and Phenotypic Divergences of SARS-CoV-2 Genes. Viruses 12:498.

Davies NG, Abbott S, Barnard RC, Jarvis CI, Kucharski AJ, Munday JD, Pearson CAB, Russell TW, Tully DC, Washburne AD, Wenseleers T, Gimma A, Waites W, Wong KLM, van Zandvoort K, Silverman JD, Group CC-W, Consortium C-GU, Diaz-Ordaz K, Keogh R, Eggo RM, Funk S, Jit M, Atkins KE, Edmunds WJ. 2021. Estimated transmissibility and impact of SARS-CoV-2 lineage B.1.1.7 in England. Science 372. 10.1126/science.abg3055

Felsenstein J. 1985. Confidence Limits on Phylogenies: An Approach Using the Bootstrap. Evolution 39:783-791.

Felsenstein J. 2005. PHYLIP (phylogeny inference package) version 3.6. Distributed by Author. 

http://evolution.genetics.washington.edu/phylip.html.

251 252 253 254 255

Furuse Y. 2021. Genomic sequencing effort for SARS-CoV-2 by country during the pandemic.

$$
\text { International Journal of Infectious Diseases 103:305-307. }
$$

Gralinski LE, Menachery VD. 2020. Return of the Coronavirus: 2019-nCoV. Viruses 12:135.

Guan Q, Sadykov M, Mfarrej S, Hala S, Naeem R, Nugmanova R, Al-Omari A, Salih S, Mutair AA, Carr MJ, Hall WW, Arold ST, and Pain A. 2020. A genetic barcode of SARS-CoV-2 for monitoring global distribution of different clades during the COVID-19 pandemic. Int J Infect Dis 100:216-223. 10.1016/j.ijid.2020.08.052

Gong Y-N, Tsao K-C, Hsiao M-J, Huang C-G, Huang P-N, Huang P-W, Lee K-M, Liu Y-C, Yang S-L, Kuo R-L, Chen K-F, Liu Y-C, Huang S-Y, Huang H-I, Liu M-T, Yang J-R, Chiu C-H, Yang C-T, Chen G-W, and Shih S-R. 2020. SARS-CoV-2 genomic surveillance in Taiwan revealed novel ORF8-deletion mutant and clade possibly associated with infections in Middle East. Emerging Microbes \& Infections 9:1457-1466. 10.1080/22221751.2020.1782271 PMID - 32543353

Isabel S, Graña-Miraglia L, Gutierrez JM, Bundalovic-Torma C, Groves HE, Isabel MR, Eshaghi A, Patel SN, Gubbay JB, Poutanen T, Guttman DS, and Poutanen SM. 2020. Evolutionary and structural analyses of SARS-CoV-2 D614G spike protein mutation now documented worldwide. Scientific Reports 10:14031. 10.1038/s41598-020-70827-z PMID - 32820179 Islam MR, Hoque MN, Rahman MS, Alam ASMRU, Akther M, Puspo JA, Akter S, Sultana M, Crandall KA, and Hossain MA. 2020. Genome-wide analysis of SARS-CoV-2 virus strains circulating worldwide implicates heterogeneity. Scientific Reports 10:14004. 10.1038/s41598-020-70812-6 PMID - 32814791 Jian MJ, Chung HY, Chang CK, Lin JC, Yeh KM, Chiu SK, Wang YH, Liao SJ, Li SY, Hsieh SS, Perng CL, Chang FY, and Shang HS. 2021. Novel automated sample-to-result SARS-CoV-2 laboratorydeveloped RT-PCR assay for high-throughput testing using LabTurbo AIO 48 system. Clin Chim Acta 514:54-58. 10.1016/j.cca.2020.12.003 Jian MJ, Chung HY, Chang CK, Hsieh SS, Lin JC, Yeh KM, Chen CW, Chang FY, Chiu SK, Hung KS, Liu MT, Yang JR, Perng CL, Shang HS. 2021. 
275 Investigation of One Familial Cluster of COVID-19 in Taiwan: Differentiation of Genetic Variation

276 Among Isolates and Implications for Epidemiological Investigation and Surveillance by Genomic

277 Assay. Infection and Drug Resistance 14:971-977. 10.2147/IDR.S298451

278 Kannan SR, Spratt AN, Quinn TP, Heng X, Lorson CL, Sönnerborg A, Byrareddy SN, and Singh K.

279 2020. Infectivity of SARS-CoV-2: there Is Something More than D614G? Journal of Neuroimmune

280 Pharmacology 15:574-577. 10.1007/s11481-020-09954-3 PMID - 32930936 Korber B, Fischer WM,

281 Gnanakaran S, Yoon H, Theiler J, Abfalterer W, Hengartner N, Giorgi EE, Bhattacharya T, Foley B,

282 Hastie KM, Parker MD, Partridge DG, Evans CM, Freeman TM, Silva TId, McDanal C, Perez LG,

283 Tang H, Moon-Walker A, Whelan SP, LaBranche CC, Saphire EO, Montefiori DC, Group SC-G,

284 Angyal A, Brown RL, Carrilero L, Green LR, Groves DC, Johnson KJ, Keeley AJ, Lindsey BB,

285

286

287

Parsons PJ, Raza M, Rowland-Jones S, Smith N, Tucker RM, Wang D, and Wyles MD. 2020.

Tracking Changes in SARS-CoV-2 Spike: Evidence that D614G Increases Infectivity of the

COVID-19 Virus. Cell. 10.1016/j.cell.2020.06.043 PMID - 32697968

288

289

Kumar S, Stecher G, Li M, Knyaz C, Tamura K. 2018. MEGA X: Molecular Evolutionary Genetics

290

Analysis across Computing Platforms. Molecular Biology and Evolution 35:1547-1549.

291

Lauring AS, Hodcroft EB. 2021. Genetic Variants of SARS-CoV-2-What Do They Mean? JAMA

325:529-531.

292 Leung K, Shum MHH, Leung GM, Lam TTY, Wu JT. 2021. Early transmissibility assessment of the

293 N501Y mutant strains of SARS-CoV-2 in the United Kingdom, October to November 2020.

$294 \quad$ Eurosurveillance 26:2002106.

295 Lin C, Braund WE, Auerbach J, Chou J-H, Teng J-H, Tu P, and Mullen J. 2020. Early Release - Policy

296 Decisions and Use of Information Technology to Fight 2019 Novel Coronavirus Disease, Taiwan -

297 Volume 26, Number 7-July 2020 - Emerging Infectious Diseases journal - CDC. Emerging

298 Infectious Diseases 26:1506-1512. 10.3201/eid2607.200574 PMID - 32228808 Lin CY, Wang WH,

299 Urbina AN, Tseng SP, Lu PL, Chen YH, Yu ML, Wang SF. 2020. Importation of SARS-CoV-2

300 infection leads to major COVID-19 epidemic in Taiwan. International Journal of Infectious

PeerJ reviewing PDF | (2021:04:59830:2:1:NEW 25 Jul 2021) 
302 Lo SW, Jamrozy D. 2020. Genomics and epidemiological surveillance. Nature Reviews Microbiology $303 \quad 18: 478.10 .1038 / \mathrm{s} 41579-020-0421-0$

304 Perng C-L, Jian M, Jr., Chang C-K, Lin J-C, Yeh K-M, Chen C-W, Chiu S-K, Chung H-Y, Wang Y-H, 305 Liao S-J, Li S-Y, Hsieh S-S, Tsai S-H, Chang F-Y, Shang H-S. 2020. Novel rapid identification of 306 Severe Acute Respiratory Syndrome Coronavirus 2 (SARS-CoV-2) by real-time RT-PCR using BD 307 Max Open System in Taiwan. PeerJ 8:e9318. 10.7717/peerj.9318

308 Rodriguez-Morales AJ, MacGregor K, Kanagarajah S, Patel D, Schlagenhauf P. 2020. Going global 309 Travel and the 2019 novel coronavirus. Travel Medicine and Infectious Disease 33:101578.

310 Sekizuka T, Kuramoto S, Nariai E, Taira M, Hachisu Y, Tokaji A, Shinohara M, Kishimoto T, Itokawa K, Kobayashi Y, Kadokura K, Kamiya H, Matsui T, Suzuki M, and Kuroda M. 2020. SARS-CoV-2 Genome Analysis of Japanese Travelers in Nile River Cruise. Frontiers in Microbiology 11:1316. 10.3389/fmicb.2020.01316 PMID - 32582136 Shu Y, McCauley J. 20170 GISAID: Global initiative on sharing all influenza data - from vision to reality. Eurosurveillance 30494. J, Thompson JD, and Higgins DG. 2011. Fast, scalable generation of high-quality protein multiple sequence alignments using Clustal Omega. Mol Syst Biol 7:539. 10.1038/msb.2011.75 Uddin M, Mustafa F, Rizvi TA, Loney T, Suwaidi HA, Al-Marzouqi AHH, Eldin AK, Alsabeeha N, Adrian TE, Stefanini C, Nowotny N, Alsheikh-Ali A, and Senok AC. 2020. SARS-CoV-2/COVID-19: Viral Genomics, Epidemiology, Vaccines, and Therapeutic Interventions. Viruses 12. 10.3390/v12050526 Vilar S, Isom DG. 2021. One Year of SARS-CoV-2: How Much Has the Virus Changed? Biology 324 Technology, and Proactive Testing. JAMA 323:1341-1342.

325 Wu Z, McGoogan JM. 2020. Characteristics of and Important Lessons from the Coronavirus Disease 2019 (COVID-19) Outbreak in China: Summary of a Report of 72314 Cases from the Chinese 
327 Center for Disease Control and Prevention. JAMA 323:1239-1242.

328 Xie M, Chen Q. 2020. Insight into 2019 novel coronavirus - An updated interim review and lessons from 329 SARS-CoV and MERS-CoV. International Journal of Infectious Disease 94:119-124.

330 Zhang L, Jackson CB, Mou H, Ojha A, Peng H, Quinlan BD, Rangarajan ES, Pan A, Vanderheiden A, 331 Suthar MS, Li W, Izard T, Rader C, Farzan M, and Choe H. 2020. SARS-CoV-2 spike-protein

332 D614G mutation increases virion spike density and infectivity. Nature Communications 11:6013.

$333 \quad 10.1038 / \mathrm{s} 41467-020-19808-4$ PMID - 33243994 


\section{Figure Legends}

335

336 Figure 1. Phylogenetic analysis of the genomes of 138 strains of SARS-CoV-2 linking the five imported 337 cases identified in the current study to previously reported genome sequences. Human coronavirus strain 338 229E (accession number AF304460.1)、HKU1 (accession number NC_006577.2)、NL63 (accession 339 number AY567487.2) and OC43 (accession number AY391777.1).

340

341 Figure 2. Haplotype network established using genome-wide single-nucleotide variations of SARS-CoV3422 isolates. The haplotype of the SARS-CoV-2 genome sequences of five strains (TSGH-04, TSGH-08, 343 TSGH-22, TSGH-23, and TSGH-29) collected from five patients localized in two clusters, which were 344 mainly comprised of isolates from North America and Europe. 


\section{Table $\mathbf{1}$ (on next page)}

Table 1. Travel history and basic case information for subjects in this study.

The table showed the travel history and basic case information for subjects in this study. 
1 Table 1. Travel history and basic case information for subjects in this study.

\begin{tabular}{|c|c|c|c|c|c|c|}
\hline Report case & Accession ID & Gender/Age & $\begin{array}{l}\text { Travel } \\
\text { History }\end{array}$ & $\begin{array}{l}\text { Collection } \\
\text { Date }\end{array}$ & $\begin{array}{l}\text { SARS- } \\
\text { CoV-2 } \\
\text { RT-PCR }\end{array}$ & $\begin{array}{l}\mathrm{Ct} \\
\text { value }\end{array}$ \\
\hline $\begin{array}{c}\text { Case } 1 \\
\text { TSGH-04 }\end{array}$ & EPI_ISL_426632 & Male/30 & USA & $03 / 20 / 2020$ & Positive & 22 \\
\hline $\begin{array}{c}\text { Case } 2 \\
\text { TSGH-08 }\end{array}$ & EPI_ISL_427395 & Female/64 & USA & $03 / 23 / 2020$ & Positive & 14 \\
\hline $\begin{array}{c}\text { Case } 3 \\
\text { TSGH-22 }\end{array}$ & EPI_ISL_436107 & Male/25 & USA & $03 / 31 / 2020$ & Positive & 17 \\
\hline $\begin{array}{c}\text { Case4 } \\
\text { TSGH-23 }\end{array}$ & EPI_ISL_436108 & Female/21 & USA & $04 / 02 / 2020$ & Positive & 23 \\
\hline $\begin{array}{c}\text { Case5 } \\
\text { TSGH-29 }\end{array}$ & EPI_ISL_447255 & Female/32 & USA & $03 / 17 / 2020$ & Positive & 26 \\
\hline
\end{tabular}

2 CT value: cycle threshold value 


\section{Table 2 (on next page)}

Table 2. Mutations in the genome sequence of five SARS-CoV-2 strains

The table 2 showed mutations in the genome sequence of five SARS-CoV- 2 strains collected from individuals with a travel history to the United States of America 
1 Table 2. Mutations in the genome sequence of five SARS-CoV-2 strains collected from individuals with

2 a travel history to the United States of America

\begin{tabular}{|c|c|c|c|c|c|}
\hline & Case 1 & Case 2 & Case 3 & Case 4 & Case 5 \\
\hline Lineage (GISAID clade) $^{\mathrm{a}}$ & \multicolumn{5}{|c|}{ GH } \\
\hline
\end{tabular}

\section{Best reference hit}

$\mathrm{NSP} 1 \mathrm{hCoV}$ -

19/Wuhan/WIV04/2019

NSP2 hCoV-

19/Wuhan/WIV04/2019

$\mathrm{NSP} 3 \mathrm{hCoV}$ -

19/Wuhan/WIV04/2019

NSP4 hCoV-

19/Wuhan/WIV04/2019

NSP5 hCoV-

19/Wuhan/WIV04/2019

NSP6 hCoV-

19/Wuhan/WIV04/2019

NSP7 hCoV-

19/Wuhan/WIV04/2019

NSP8 hCoV-

19/Wuhan/WIV04/2019

NSP9 hCoV-

19/Wuhan/WIV04/2019

$\mathrm{NSP} 10 \mathrm{hCoV}$ -

19/Wuhan/WIV04/2019

NSP11 hCoV-

19/Wuhan/WIV04/2019

$\mathrm{NSP} 12 \mathrm{hCoV}$ -

19/Wuhan/WIV04/2019

$\mathrm{NSP} 13 \mathrm{hCoV}-$

19/Wuhan/WIV04/2019

$\mathrm{NSP} 14 \mathrm{hCoV}$ -

19/Wuhan/WIV04/2019

\begin{tabular}{|c|c|c|c|c|}
\hline$-b$ & - & - & - & - \\
\hline T85I & T85I & T85I & T85I & - \\
\hline- & P1403S & - & - & $\begin{array}{l}\text { F1141X, } \\
\text { R1449G }\end{array}$ \\
\hline- & - & - & - & - \\
\hline- & - & - & - & - \\
\hline- & - & - & - & A161S, I266L \\
\hline- & S25L & V58F & - & - \\
\hline- & - & - & - & - \\
\hline- & - & - & - & - \\
\hline - & - & - & - & - \\
\hline - & - & - & - & - \\
\hline P323L & P323L & P323L & P323L & - \\
\hline - & - & - & - & M436L \\
\hline L177F & A320V & - & - & - \\
\hline
\end{tabular}




\begin{tabular}{|c|c|c|c|c|c|}
\hline $\begin{array}{l}\text { NSP15 hCoV- } \\
\text { 19/Wuhan/WIV04/2019 }\end{array}$ & - & - & - & - & - \\
\hline $\begin{array}{l}\text { NSP16 hCoV- } \\
\text { 19/Wuhan/WIV04/2019 }\end{array}$ & - & - & - & - & - \\
\hline $\begin{array}{l}\text { Spike hCoV- } \\
\text { 19/Wuhan/WIV04/2019 }\end{array}$ & D614G & D614G & D614G & D614G & - \\
\hline NS3 hCoV-19/Wuhan/WIV04/2019 & Q57H & Q57H & Q57H & Q57H & - \\
\hline E hCoV-19/Wuhan/WIV04/2019 & - & - & - & - & - \\
\hline M hCoV-19/Wuhan/WIV04/2019 & - & - & - & - & I8S \\
\hline NS6 hCoV-19/Wuhan/WIV04/2019 & - & - & - & - & - \\
\hline $\begin{array}{l}\text { NS7a hCoV- } \\
\text { 19/Wuhan/WIV04/2019 }\end{array}$ & - & - & - & - & - \\
\hline $\begin{array}{l}\text { NS7b hCoV- } \\
\text { 19/Wuhan/WIV04/2019 }\end{array}$ & - & - & - & - & - \\
\hline NS8 hCoV-19/Wuhan/WIV04/2019 & - & - & - & - & - \\
\hline N hCoV-19/Wuhan/WIV04/2019 & - & - & S183Y & - & - \\
\hline
\end{tabular}

3 a Clade and lineage nomenclature was developed by Sebastian Maurer-Stroh et al, based on marker

4 mutations used in GISAID

$5 \quad \mathbf{b}$ no mutations

6

7 
Figure 1

Phylogenetic analysis of the genomes

Figure 1. Phylogenetic analysis of the genomes of 42 strains of SARS-CoV-2 linking the five imported cases of the current study to previously reported genome sequences. 


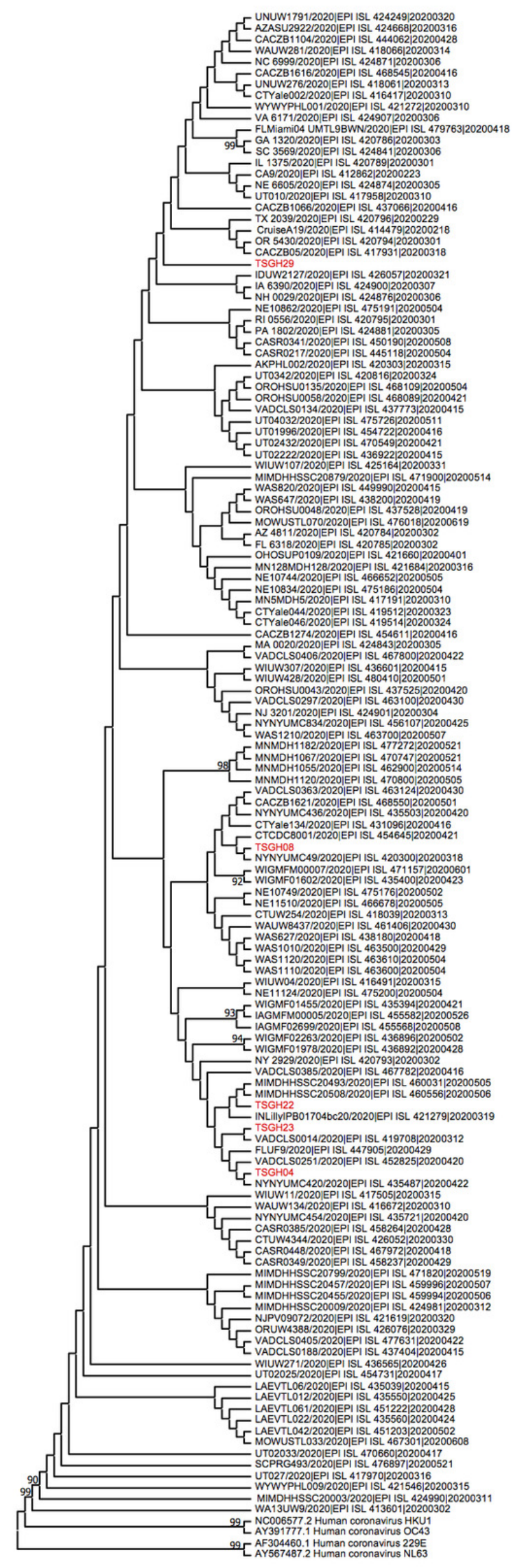


Figure 2

Haplotype network established using genome-wide single-nucleotide variations of SARSCoV-2 isolates

Figure 2. Haplotype network established using genome-wide single-nucleotide variations of SARS-CoV-2 isolates. The haplotype of the SARS-CoV-2 genome sequences for five strains (TSGH-04, TSGH-08, TSGH-22, TSGH-23, and TSGH-29) isolated from five different patients was found to localize in two clusters, which were mainly comprised by North America and European isolates.

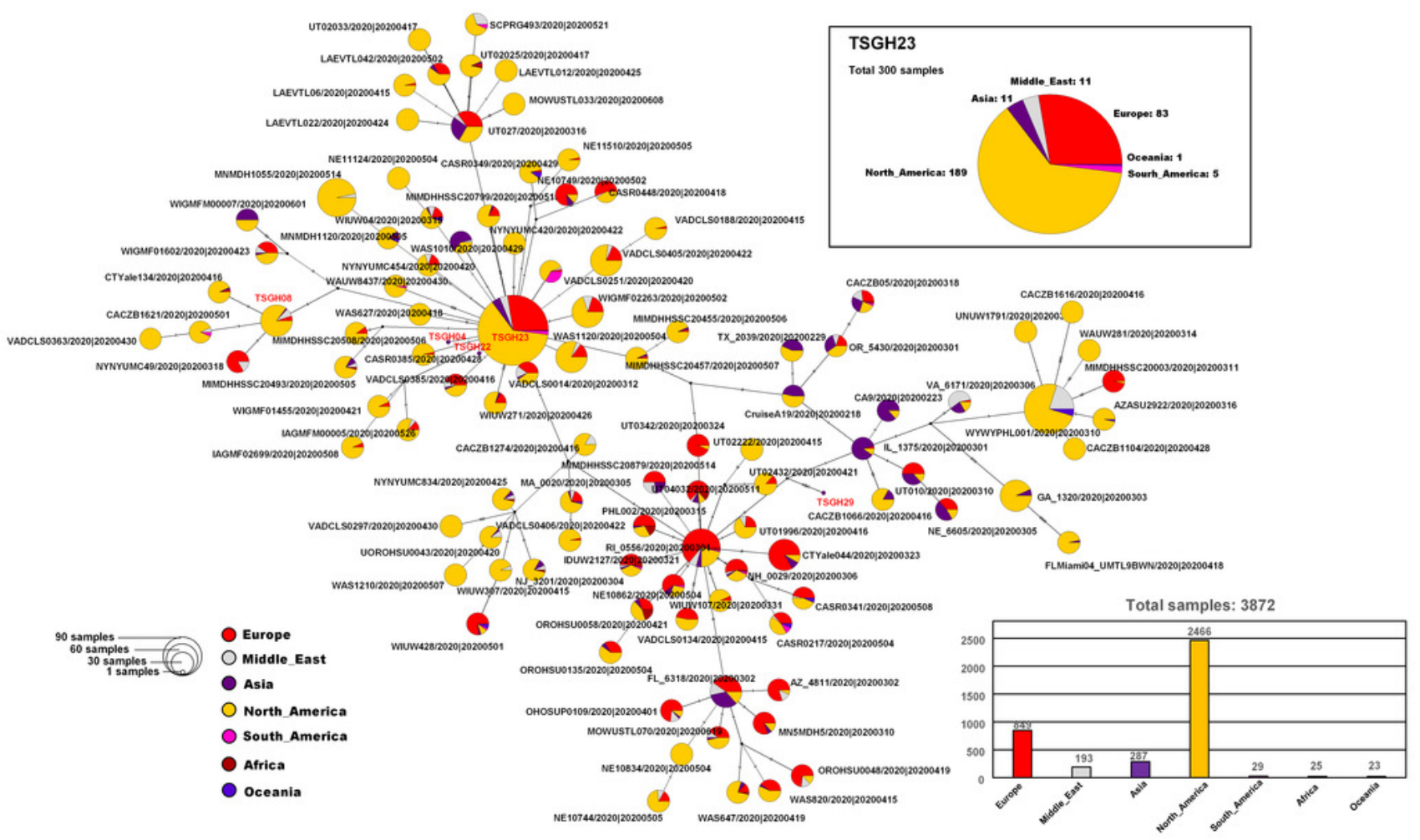

